\title{
Appendix A: Transcription Conventions
}

\begin{tabular}{|c|c|}
\hline . & sentence final falling intonation \\
\hline , & clause-final intonation (“more to come") \\
\hline$!$ & animated tone \\
\hline$?$ & rising intonation (not necessarily a question) \\
\hline- & glottal stop: sound abruptly cut off; self-interruption \\
\hline italics & emphatic stress \\
\hline CAPS & spoken much louder than surrounding text \\
\hline${ }^{\circ}$ words ${ }^{\circ}$ & spoken more quietly than surrounding text \\
\hline : & after a vowel indicates elongated vowel sound \\
\hline$::$ & more elongation \\
\hline /words/ & in slashes indicate uncertain transcription \\
\hline $\begin{array}{l}\text { wo[rds } \\
\text { [words }\end{array}$ & $\begin{array}{l}\text { beginning of overlapping speech, right brackets may be used to indicate the } \\
\text { end of the overlap }\end{array}$ \\
\hline$=$ & latching \\
\hline hhh & aspiration \\
\hline .hhh & inhale \\
\hline $\mathrm{HHH}$ & aspiration/laughter while speaking \\
\hline (sarcastically) & description of voice quality or non-verbal action \\
\hline (...) & part of a turn or some intervening turns at talk have been omitted \\
\hline (.) & pause of less than one second \\
\hline (7) & pause of this many seconds \\
\hline “words" & speaker is quoting another person or adopting his/her voice \\
\hline [fəneDlk] & phonetic transcription \\
\hline -) great $\odot$ & smiling voice quality \\
\hline
\end{tabular}

